\title{
Rivastigimine for Relatively Younger Alzheimer's Disease Patient
}

\author{
Kentaro Horiuchi ${ }^{1}$, Koji Hori $^{1^{*}}$, Misa Hosoi ${ }^{1}$, Kimiko Konishi ${ }^{1,2}$, Hiroi Tomioka ${ }^{1}$ and Mitsugu Hachisu ${ }^{3}$ \\ ${ }^{1}$ Department of Psychiatry, Showa University Northern Yokohama Hospital, Kanagawa, Japan \\ ${ }^{2}$ Tokyo Metropolitan Tobu Medical Center for Persons With Developmental/Multiple Disabilities, Tokyo, Japan \\ ${ }^{3}$ Department of Clinical Pharmacology, School of Pharmaceutical Sciences, Showa University, Tokyo, Japan \\ "Corresponding author: Koji Hori, Department of Psychiatry, Showa University Northern Yokohama Hospital, 35-1 Chigasakichuo, Tsuzukiku, Yokohama-City, \\ Kanagawa, 224-8503, Japan, Tel: +81-45-949-7000; Fax: +81-45-949-7927; E-mail: kojihori@med.showa-u.ac.jp
}

Received date: May 09, 2014, Accepted date: June 16, 2014, Published date: June 27, 2014

Copyright: $\odot 2014$ Hiruichi K, et al. This is an open-access article distributed under the terms of the Creative Commons Attribution License, which permits unrestricted use, distribution, and reproduction in any medium, provided the original author and source are credited.

\begin{abstract}
We presented the patient with relative younger Alzheimer's disease (AD) whose clinical symptoms and cognitive functions were responsive not to donepezil but to rivastigmine. Age of initial visit our memory clinic of this patient was relatively younger. It is considered that in relative older patient both $A D$ pathology and aging cause cognitive dysfunctions. However, in relative younger patient not aging but only AD pathology causes cognitive dysfunctions. Therefore, $A D$ pathology was thought to be more pronounced in our patient than relatively older patients with same cognitive disturbances. In AD, glia cells and amyloids proliferate and nervous cells shrink. Butyrylcholinesterase (BuChE) exists in glia cells and amyloids. Therefore, when AD progresses, acetylcholinesterase (AChE) decreases and BuChE increases. Accordingly the ratio of BuChE/AChE increases. Therefore, when at mild stage such our patient, not donepezil but rivastigmine which has inhibiting actions on both AChE and BuChE was suitable.
\end{abstract}

Keywords: Alzheimer's disease; Butyrylcholinesterase; Rivastigmine; Relative younger patient

\section{Introduction}

In Japan, two cholinesterase inhibitors (ChEIs), galantamine and rivastigmine, are marketed in 2011 in addition to donepezil. As one $\mathrm{ChEI}$ is not allowed to be combined with other ChEI, we should select one of three ChEIs for the patient Alzheimer's disease (AD). Among the ChEIs, rivastigmine is unique in its characteristics to inhibit not only acetylcholinesterase (AChE) but also butyrylcholinesterase (BuChE) [1]. Therefore, this action causes more potent upregulation of acetylcholine (ACh). Here we report a case of an $\mathrm{AD}$ patient showing amelioration by rivastigmine. Informed consent of reporting was obtained by this patient and his wife.

\section{Case Presentations}

Patient was a 69-year-old male patient who came to the memory clinic of our hospital complaining of memory deterioration. He had been experiencing amnesia 4 years age. Then he could not manage his finance 2 years age. He showed apathy, irritability and temporal disorientation 1 year before. On initial examination at our clinic, the patient's Mini-Mental State Examination (MMSE) [2] score was 20 and magnetic resonance imaging (MRI) showed periventricular high density, however, there was no prominent atrophy in his brain. We diagnosed our patient with mild $\mathrm{AD}$ [3] because his cognitive function was mildly deteriorated, and he complained of memory disturbances and apathy. We prescribed donepezil at $5 \mathrm{mg}$ daily and soon after starting this treatment, his memory disturbances and apathy were ameliorated. However, he showed apathy again. Seven months later, his MMSE score was down to 19 . We considered that donepezil was not effective because his wife complained that his demented state was not changed. We prescribed rivastigmine instead of donepezi. Five months later, his MMSE score became 25. His wife said that the progression of his dementia became slow and his apathy was ameliorated. At 2 years from first visit, his MMSE score became 27. At that time, his wife said that he was vivid and his memorial disturbance was ameliorated, i.e. rivastigmine was also effective.

\section{Discussion}

We considered the patient with relative younger $\mathrm{AD}$ was responsive to not donepezil but rivastigmine because his clinical symptoms especially apathy were ameliorated and his cognitive function was improved with the prescription of rivastigmine instead of donepezil. The head to head evidence for the comparative efficacy of donepezil and rivastigmine in $\mathrm{AD}$ is limited to 2 clinical trials [4,5]. Those showed the similar effects on cognitive functions, however, Bullock et al. [4] reported that rivastigmine showed a statistically significant advantage on measures of activities of daily living and global functions and that $\mathrm{AD}$ patients who were below 75 years old and had symptoms suggestive of concomitant Lewy body disease showed significantly greater benefits from rivastigmine treatment. Wilkinson et al. [5] reported that donepezil was better tolerated with fewer discontinuations due to adverse effects than rivastigmine. From these results we should consider individual variations. From this point of view, as Bullock et al. reported, relative younger patients might be responsive to rivastigmine than donepezil.

Main finding of neurotransmitter change in $\mathrm{AD}$ is downregulation of ACh [6]. The downregulation of ACh is related to cognitive dysfunctions such as memory disturbance and disorientations [3,7]. However, it is considered that $\mathrm{ACh}$ also regulates inflammatory system, i. e., referred to as antiinflammatory pathway $[8,9]$. Therefore, we speculated that some cytokines caused by upregulations of inflammations have anticholinergic activity (AA), which accelerated $\mathrm{AD}$ pathology (amyloids and tau) [10-12]. Generally speaking, AD progresses more rapidly in moderate stage than in mild stage [13]. We also speculated that when downregulations of $\mathrm{ACh}$ reached critical 
level, AA generated by upregulations of inflammations accelerated AD pathology [12]. Therefore, it is important not to downregulate ACh so as not to generate $\mathrm{AA}$ and not to accelerate $\mathrm{AD}$ pathology.

In normal brain, main enzyme degrading $\mathrm{ACh}$ is $\mathrm{AChE}$ [14], which exists in nervous cells. However, in $\mathrm{AD}$, glia cells and amyloids proliferate and nervous cells shrink. BuChE exists in glia cells and amyloids [15]. Therefore, when AD progresses, AChEs decrease and BuChEs increase. Accordingly the ratio of BuChEs/AChEs increases. In MCI stage, even when $\mathrm{AD}$ pathology is burdened, nervous cells are almost normal. Therefore, main enzyme which catalyses ACh is AChE. However, in mild stage in $\mathrm{AD}$, when nervous cells decrease and glia cells and amyloids increase, main enzymes those catalyse ACh are not only AChE but also BuChE. In fact Perry et al. reported that when AD progresses, AChEs decrease with liner fashion and BuChEs increase with sigmoid fashion [16]. Moreover, $\mathrm{BuChE}$ is considered to accelerate the numbers of amyloids, i.e., BuChE is considered to accelerate AD pathology [17]. Therefore, when at mild stage such as our patient, not donepezil but rivastigmine which has inhibiting actions on both $\mathrm{AChE}$ and $\mathrm{BuChE}$ was suitable.

Moreover, age of initial visit to our memory clinic of our patient was relative younger. It is considered that in relative older patient both $\mathrm{AD}$ pathology and aging cause cognitive dysfunctions [16]. However, in relative younger patient not aging but only $\mathrm{AD}$ pathology causes cognitive dysfunctions. Therefore, $\mathrm{AD}$ pathology was thought to be more pronounced in our patient than relative older patients with same cognitive disturbances. There might be a possibility that the lost potency of donepzil might result from drug resistance due to repetitive usage, however, it might not happen to rivastigmine with prolonged treatment because rivastigmine showed efficacy for 1 year and 5 months.

We considered that from these three points of view, our patient was responsive not to donepezil but to rivastigmine and that when relatively younger $\mathrm{AD}$ patient with at mild stage receive the treatment of $\mathrm{AD}$, not donepezil but rivastigmine which has inhibiting actions to both AChE and BuChE was suitable.

\section{Conflicts of Interest}

Koji Hori received lecture fee from Eisai Co., Ltd., Pfizer Japan Inc., Novartis Pharma K.K., Daiichi Sankyo Inc., Ono Pharmacuetical Co., Ltd., Janssen Pharmaceutical K.K., Yoshitomi Yakuhin Co. and Mitsubishi Tanabe Pharma Co. Mitsugu Hachisu received fund from Astellas Pharma Inc., Meiji Seika Pharma Co., Ltd., Dainippon Sumitomo Pharm Co., Ltd., Eli Lilly Japan K.K. and Shionogi \& Co., Ltd. and received lecture fee from Meiji Seika Pharma Co., Ltd. and Mitsubishi Tanabe Pharma Co.

\section{Acknowledgement}

Funding for this study was received from Eisai Co., Ltd., Daiichi Sankyo Inc. and Ono Pharmacuetical Co., Ltd.

\section{References}

1. Ogura H, Kosasa T, Kuriya Y, Yamanishi Y (2000) Comparison of inhibitory activities of donepezil and other cholinesterase inhibitors on acetylcholinesterase and butyrylcholinesterase in vitro. Methods Find Exp Clin Pharmacol 22: 609-613.

2. Folstein MF, Folstein SE, McHugh RP (1975) "Mini-Mental State." A practical method for grading the cognitive state of patients for the clinician. J Psy Res 12: 189-198.

3. McKhann G, Drachman D, Folstein M, Katzman R, Price D, et al. (1984) Clinical diagnosis of Alzheimer's disease: report of the NINCDS-ADRDA Work Group under the auspices of Department of Health and Human Services Task Force on Alzheimer's Disease. Neurology 34: 939-944.

4. Bullock R, Touchon J, Bergman H, Gambina G, He Y, et al. (2005) Rivastigmine and donepezil treatment in moderate to moderately-severe Alzheimer's disease over a 2-year period. Curr Med Res Opin 21: 1317-1327.

5. Wilkinson DG, Passmore AP, Bullock R, Hopker SW, Smith R, et al. (2002) A multinational, randomised, 12-week, comparative study of donepezil and rivastigmine in patients with mild to moderate Alzheimer's disease. Int J Clin Pract 56: 441-446.

6. Whitehouse PJ, Price DL, Struble RG, Clark AW, Coyle JT, et al. (1982) Alzheimer's disease and senile dementia: loss of neurons in the basal forebrain. Science 215: 1237-1239.

7. Cushman LA, Stein K, Duffy CJ (2008) Detecting navigational deficits in cognitive aging and Alzheimer disease using virtual reality. Neurology 71 : 888-895.

8. Mabley JG, Pacher P, Szabo C (2009) Activation of the cholinergic antiinflammatory pathway reduces ricin-induced mortality and organ failure in mice. Mol Med 15: 166-172.

9. Nazarov PG, Krylova IB, Evdokimova NR, Nezhinskaya GI, Butyugov AA (2007) C-reactive protein: a pentraxin with anti-acetylcholine activity. Life Sci 80: 2337-2341.

10. Hori K, Konishi K, Akita R (2013) Proposal of endogenous anticholinergic hypothesis in Alzheimer's dsease, Jpn J Neuropsychopharmacol 33: 117-126.

11. Hori K, Konishi K, Akashi N, Timioka H, Akita R, et al. (2014) Serum anticholinergic activity: A possible peripheral marker of the anticholinergic burden in the central nervous system in Alzheimer's disease. Disease Markers 3: Article ID 459013.

12. Hori K, Konishi K, Tani M, Timioka H, Akita R, et al. (2014) Why does the progression of Alzheimer's disease accelerate? Ann Psychiatry Ment Health. 2: 1006.

13. Ji M, Xiong C, Grundman M (2003) Hypothesis testing of a change point during cognitive decline among Alzheimer's disease patients. J Alzheimers Dis 5: 375-382.

14. Arendt T, Brückner MK, Lange M, Bigl V (1992) Changes in acetylcholinesterase and butyrylcholinesterase in Alzheimer's disease resemble embryonic development--a study of molecular forms. Neurochem Int 21: 381-396.

15. Darvesh S, Hopkins DA, Geula C (2003) Neurobiology of butyrylcholinesterase. Nat Rev Neurosci 4: 131-138.

16. Perry EK, Tomlinson BE, Blessed G, Bergmann K, Gibson PH, et al. (1978) Correlation of cholinergic abnormalities with senile plaques and mental test scores in senile dementia. Br Med J 2: 1457-1459.

17. Greig NH, Utuki T, Iguram D, Wang Y, Pepeu G, et al. (2005) Selective butyrylcholinesterase inhibition elevates brain acetylcholine, augments learning and lowers Alzheimer $\beta$-amyloid peptide in rodent. PNAS 102: 17213-17218. 